\title{
The style of nursing leadership in hospitals is an independent risk factor for 30-day mortality of patients in acute care
}

\section{Lisbeth Fagerström}

$10.1136 / \mathrm{ebn} 1140$

Buskerud University College, Drammen, Norway

Correspondence to: Lisbeth Fagerström Buskerud University College, Department of Health Sciences, Papirbredden, Grønland 58, 3045 Drammen, Norway; lisbeth.fagerstrom@hibu.no

\section{Commentary on: Cummings GG, Midodzi WK, Wong CA, et al. The contribution of hospital nursing leadership styles to 30-day patient mortality. Nurs Res 2010;59:331-9.}

\section{Context}

The focus of the study is on nursing leadership and its association to patient mortality. Cummings and colleagues state in the introduction that the relationship between nursing leadership styles in hospitals and patient outcomes has not been explored in many studies. The authors discuss the influence of different nursing leadership styles, such as task- and relationship-focused, emotionally intelligent and transformational leadership styles.

\section{Methods}

The purpose of the study was to examine the contribution of hospital nursing leadership styles to 30-day mortality after controlling for patient demographics, comorbidities and hospital factors. Secondary data about nurses, patients and institutions from 90 hospitals collected from 1998 to 1999 through the Canadian Alberta Nurse Survey were used. The main analysis method was hierarchical logistic regression analyses with logit link. The effect of nursing leadership, patient factors and hospital nursing variables on mortality was estimated, including their independent effect after adjustment. Finally, the residual effect of leadership styles was assessed after controlling for all patients, hospital nursing and institutional factors.

\section{Findings}

After controlling for patient demographics, comorbidities and institutional and hospital characteristics, nursing leadership styles explained $5.1 \%$ of the $72.2 \%$ variance in mortality across the hospitals. High-resonant leadership (eg, a style that is visionary, coaching, affiliative and democratic) was significantly related to lower mortality.

\section{Commentary}

The strengths of the article are a well-structured literature review including the most important factors/issues associated with outcome variables in healthcare; it is a comprehensive empirical study based on large data material. The chosen variables of the tested model are generally described in a clear manner. To increase trustworthiness, some nursing predictor variables such as nursing time, skill mix and nurse education could have been described in greater detail. Furthermore, the principle used to include hospitals in the study is debatable; the criterion for inclusion was that at least five registered nurses had responded. Can so few responses give an accurate picture of leadership styles?

The study results are based on data that is more than 10 years old. Patient mortality has historically been used as the most common outcome variable in healthcare. Today, however, there are several fundamental nursing sensitive outcome indicators that could be used to effectively demonstrate that nurses comprise a critical, costeffective difference in providing safe and high-quality patient care. Workload, nursing intensity and unattended patients' needs are organisational factors that significantly affect the quality of care. ${ }^{1}$ By assessing nursing time in relation to patients' care needs, a measure of nursing intensity is achieved. When nurses' workload is at an optimal level of nursing intensity, realistic conditions for receiving good care as a patient exist. ${ }^{2}$ Nursing time as an organisational factor must be related to patients' actual needs, and the resource allocation of staff should follow patients' continuously varying needs. More detailed and reliable information regarding patients' needs, nursing intensity and nurses' workload, enabling the analysis of the effect on outcome variables, should be included in future research.

\section{Implications for practice}

The study has shown that 'nursing leadership makes a difference' for staff as well as for patients. The results indicate that more focus on leadership and management in nursing education is needed at master's as well as doctoral levels. The most characteristic features of healthcare today may be the continuously changing environment and resulting organisational changes. ${ }^{3}$ Especially during times of organisational change, the importance of clear objectives, vision, information, dialogue and leader support increases. Contextual changes to hospitals and the effect of situational leadership should be included in future research on leadership styles and outcomes. An organisation's culture is important and affects leadership style during change; the same applies to nurses' expectations of their nurse leaders. A person-centred nursing culture promotes the outcome of nursing to patients benefit and also increases nurses' well-being and job satisfaction.

\section{Competing interests None.}

\section{References}

1. Whitman GR, Kim Y, Davidson LJ, et al. The impact of staffing on patient outcomes across specialty units. J Nurs Adm 2002;32:633-9.

2. Fagerström L, Rauhala A. Benchmarking in nursing care by the RAFAELA patient classification system - a possibility for nurse managers. J Nurs Manag 2007;15:683-92.

3. Fagerström L, Salmela S. Leading change: a challenge for leaders in Nordic health care. J Nurs Manag 2010;18:613-17. 